\title{
Strong electronic correlation and strain effects at the interfaces between polar and nonpolar complex oxides
}

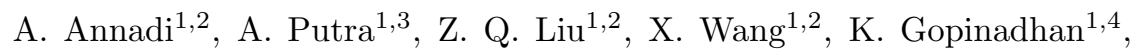 \\ Z. Huang ${ }^{1}$, S. Dhar ${ }^{1,4}$, T. Venkatesan ${ }^{1,2,4}$, and Ariando ${ }^{1,2 *}$ \\ ${ }^{1}$ NUSNNI-Nanocore, National University of Singapore, 117411 Singapore \\ ${ }^{2}$ Department of Physics, National University of Singapore, 117542 Singapore \\ ${ }^{3}$ Department of Engineering Science Programme, \\ National University of Singapore, $11^{77} 5^{r 6}$ Singapore and \\ ${ }^{4}$ Department of Electrical and Computer Engineering, \\ National University of Singapore, 117576 Singapore
}

\begin{abstract}
The interface between the polar $\mathrm{LaAlO}_{3}$ and nonpolar $\mathrm{SrTiO}_{3}$ layers has been shown to exhibit various electronic and magnetic phases such as two dimensional electron gas, superconductivity, magnetism and electronic phase separation. These rich phases are expected due to the strong interplay between charge, spin and orbital degree of freedom at the interface between these complex oxides, leading to the electronic reconstruction in this system. However, until now all of these new properties have been studied extensively based on the interfaces which involve a polar $\mathrm{LaAlO}_{3}$ layer. To investigate the role of the $\mathrm{A}$ and $\mathrm{B}$ cationic sites of the $\mathrm{ABO}_{3}$ polar layer, here we study various combinations of polar/nonpolar oxide $\left(\mathrm{NdAlO}_{3} / \mathrm{SrTiO}_{3}, \mathrm{PrAlO}_{3} / \mathrm{SrTiO}_{3}\right.$ and $\left.\mathrm{NdGaO}_{3} / \mathrm{SrTiO}_{3}\right)$ interfaces which are similar in nature to $\mathrm{LaAlO}_{3} / \mathrm{SrTiO}_{3}$ interface. Our results show that all of these new interfaces can also produce 2DEG at their interfaces, supporting the idea that the electronic reconstruction is the driving mechanism for the creation of the 2DEG at these oxide interfaces. Furthermore, the electrical properties of these interfaces are shown to be strongly governed by the interface strain and strong correlation effects provided by the polar layers. Our observations may provide a novel approach to further tune the properties of the 2DEG at the selected polar/nonpolar oxide interfaces.
\end{abstract}

PACS numbers: 73.40.Rw, 73.50.Gr, 73.20.Hb

\section{INTRODUCTION}

Heterostructures constructed from polar/nonpolar oxides, especially the $\mathrm{LaAlO}_{3} / \mathrm{SrTiO}_{3}$ interface, have been a topic of research in recent years in which along with 2DEG, novel properties have been reported [1-5]. Further, it has been demonstrated that the properties at the $\mathrm{LaAlO}_{3} / \mathrm{SrTiO}_{3}$ interface can be manipulated in many ways such as electric field effect [2], AFM charge writing [6] and adsorbates capping [7], showing the ability to tune the interface by external parameters. Moreover, in $\mathrm{LaAlO}_{3} / \mathrm{SrTiO}_{3}$ heterostructure the combined effects of lattice mismatch $(\sim 2.3 \%)$ between the $\mathrm{LaAlO}_{3}$ and $\mathrm{SrTiO}_{3}$ and electronic effects provided by polar $\mathrm{LaAlO}_{3}$ at the interface play a strong role in controlling the interface properties. Recently, C. W. Bark et al.[8] demonstrated strain effects on properties of 2DEG at the $\mathrm{LaAlO}_{3} / \mathrm{SrTiO}_{3}$ interface by growing the heterostructure on different substrates. In addition to this, electrostriction and electromechanical response were demonstrated at the $\mathrm{LaAlO}_{3} / \mathrm{SrTiO}_{3}$ heterostrures $[9,10]$. Recently, a similar 2DEG is also shown to exist at the $\mathrm{LaGaO}_{3} / \mathrm{SrTiO}_{3}$ interface [11]. In all above observations, the polar nature of the over layer is critical. The significance of the polar over layer can be further explored by investigating various new combinations of polar/nonpolar oxide heterostructures. In this scenario, a choice of variety in chemical nature and lattice structure of polar layers enable us to tune the interface properties.
Moreover this approach may help in revealing key issues such as driving mechanism of the formation of $2 \mathrm{DEG}$ which is widely believed to be originating from polarization catastrophe [12] due to this polar/nonpolar nature of the interface and the localization of carriers at this interface due to the lifting of the degeneracy of the Ti $d$ states. In this article, we investigate various combinations of polar/nonpolar oxide interfaces (Fig. 1(a)) $\mathrm{REBO}_{3} / \mathrm{SrTiO}_{3}$ (100) $(\mathrm{RE}=\mathrm{La}, \mathrm{Pr}, \mathrm{Nd}, \mathrm{B}=\mathrm{Al}, \mathrm{Ga})$ and study their structural and electrical properties. The choice of polar oxides is made in such a way that all of them are similar to the $\mathrm{LaAlO}_{3}$ in nature, having $(\mathrm{AO})^{+1},\left(\mathrm{BO}_{2}\right)^{-1}$ polar charge layers alternatively along (100) direction.

\section{EXPERIMENTAL}

Samples were prepared by pulsed laser deposition, ablating $\mathrm{REBO}_{3}$ targets onto $\mathrm{TiO}_{2}$ terminated $\mathrm{SrTiO}_{3}$ (100) substrates. Atomically flat $\mathrm{TiO}_{2}$ terminated $\mathrm{SrTiO}_{3}(100)$ substrates were obtained by employing well established conditions; buffer HF treatment for $30 \mathrm{sec}$ followed by thermal annealing at $950^{\circ} \mathrm{C}$ for 2 hours $[13,14]$. Samples were prepared in a range of oxygen partial pressure $\left(P_{\mathrm{O} 2}\right)$ of $1 \times 10^{-3}$ to $1 \times 10^{-5}$ Torr at $800^{\circ} \mathrm{C}$. The laser $(248 \mathrm{~nm})$ energy density was $1.4 \mathrm{~J} / \mathrm{cm}^{2}$ and repetition rate was $1 \mathrm{~Hz}$. During deposition, the film growth and number of unit cells were monitored by in-situ reflection high energy electron diffraction (RHEED). Samples with 
(a)

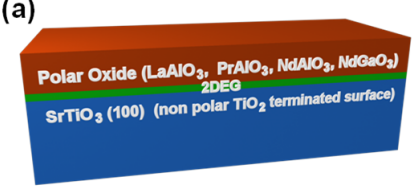

(c)

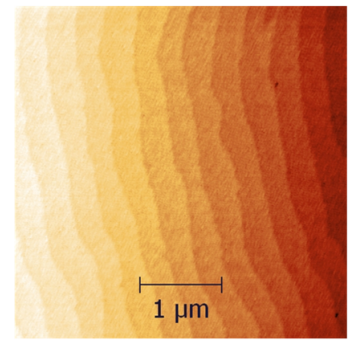

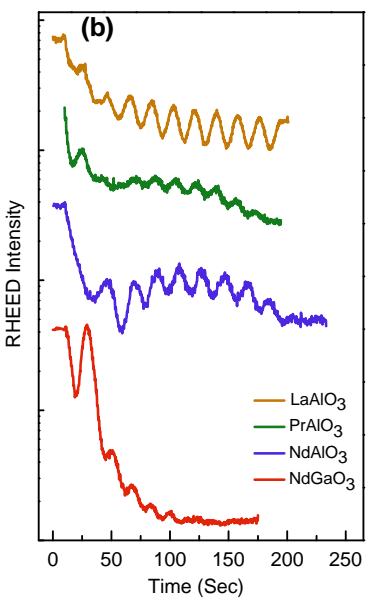

FIG. 1: (a) Schematic representation of polar/nonpolar $\mathrm{REBO}_{3} / \mathrm{SrTiO}_{3}$ (RE-Rear Earth element) heterostructure. (b) Typical RHEED oscillations obtained for the growth of different polar layers on top of $\mathrm{TiO}_{2}$ terminated $\mathrm{SrTiO}_{3}$ substrate. (c) Atomic force microscopy (AFM) image of $10 \mathrm{uc}$ $\mathrm{NdAlO}_{3} / \mathrm{SrTiO}_{3}$ sample showing an atomically smooth surface.

thicknesses ranging from 1-14 unit cells (uc) were grown for $\mathrm{NdAlO}_{3}$ while a fixed 10 uc was used for all other polar layers for comparison. Epitaxial growth of films on $\mathrm{SrTiO}_{3}$ substrates were further confirmed by high resolution X-ray diffraction (HRXRD). Electrical contacts were made with an $\mathrm{Al}$ wire-bonding directly to the interface. Sheet resistance and Hall Effect measurements were carried out to extract carrier density, mobility and electrical properties. Figure 1(b) shows RHEED intensity patterns obtained during the growth of the samples, clearly showing the layer by layer growth mode for various polar oxide layers on $\mathrm{SrTiO}_{3}$. Figure 1(c) shows AFM topography image after the growth of $10 \mathrm{uc} \mathrm{NdAlO}_{3}$ on $\mathrm{SrTiO}_{3}$ with preserved steps further confirms the layer by layer growth. Even though all these polar oxides offer substantial lattice mismatch with $\mathrm{SrTiO}_{3}$ substrate, we have successfully grown them by optimizing deposition conditions.

\section{RESULTS AND DISCUSSION}

We first discuss the case of $\mathrm{NdAlO}_{3} / \mathrm{SrTiO}_{3}$ interface. Figure 2(a) shows the dependence of sheet resistance, $R_{s}$, with temperature for the $\mathrm{NdAlO}_{3} / \mathrm{SrTiO}_{3}$ interface prepared in the $P_{O 2}$ range of $1 \times 10^{-3}-1 \times 10^{-5}$ Torr. While all samples clearly exhibit a conducting property at their interface, the high $P_{O 2}$ grown samples show strong upturns in $R_{s}$ at lower temperatures. Interestingly, even the sample grown at $P_{O 2}$ of $1 \times 10^{-4}$ Torr shows an upturn. It is noted here that in the case of $\mathrm{LaAlO}_{3} / \mathrm{SrTiO}_{3}(100)$ interfaces such an upturn emerges only for higher $P_{O 2}$ $\left(\geq 10^{-3}\right.$ Torr $)$ and "thicker" samples $(\geq 15 \mathrm{uc})[4,15]$. However, the sample grown at $P_{O 2} 1 \times 10^{-5}$ Torr showed
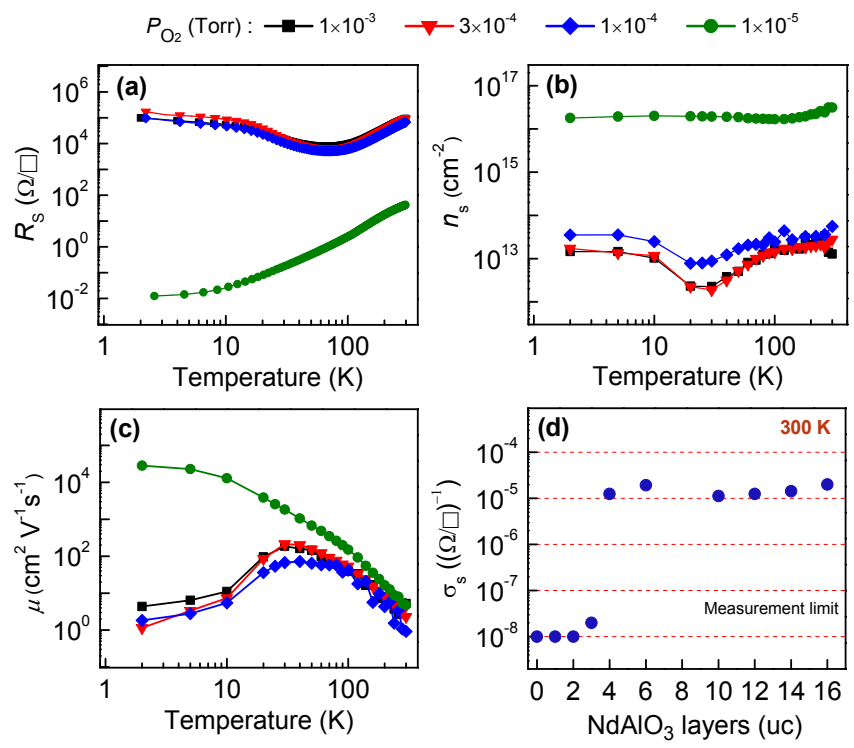

FIG. 2: (a) Temperature dependence of the sheet resistance, $R_{s}(T)$, of the $\mathrm{NdAlO}_{3} / \mathrm{SrTiO}_{3}$ interfaces, for different oxygen partial pressures $\left(P_{\mathrm{O} 2}\right)$ during growth. Temperature dependence of (b) carrier density $n_{s}$ and (c) Hall mobility $\mu$ of the $\mathrm{NdAlO}_{3} / \mathrm{SrTiO}_{3}$ interfaces. (d) Dependence of the sheet conductivity on the number of $\mathrm{NdAlO}_{3}$ unit cells for the $\mathrm{NdAlO}_{3} / \mathrm{SrTiO}_{3}$ interfaces.

higher conductivity which can be understood in terms of the presence of oxygen vacancies at the interface. Figures 2(b) and 2(c) show temperature dependence of carrier density, $n_{s}$, and mobility, $\mu$, for the $\mathrm{NdAlO}_{3} / \mathrm{SrTiO}_{3}$ samples, respectively. For high $P_{O 2}$ samples, $n_{s}(300 \mathrm{~K})$ is of the order of $4-5 \times 10^{13} \mathrm{~cm}^{-2}$. Whereas for sample grown at $1 \times 10^{-5}$ Torr, $n_{s}(300 \mathrm{~K})$ is of the order of $10^{16} \mathrm{~cm}^{-2}$, further confirming the presence of oxygen vacancies which is a common observation for the interfaces grown at lower $P_{\mathrm{O}_{2}}[16,17]$. A large carrier freeze-out towards low temperatures is observed in high $P_{O 2}$ samples. This kind of behaviour was previously observed in the cases of $\mathrm{LaAlO}_{3} / \mathrm{SrTiO}_{3}$ [18]. For all the samples, $\mu(300 \mathrm{~K})$ is of the order of $1-8 \mathrm{~cm}^{2} \mathrm{~V}^{-1} \mathrm{~s}^{-1}$ comparable to those reported for the $\mathrm{LaAlO}_{3} / \mathrm{SrTiO}_{3}$ (100) interfaces $[15,19]$. The $\mu$ follows $T^{-2}$-like dependence in the temperature range of 300-100 K, typical behaviour of Fermi liquid. However, a significant difference in $\mu$ is observed at low temperatures between samples grown at high and low $P_{O 2}$. While an abrupt drop in $\mu$ towards low temperatures is observed (which generally occurs when strong localization/magnetic scattering effects present in the system) for high $P_{O 2}$ samples, an increase in $\mu$ throughout the temperature range down to $2 \mathrm{~K}$ is observed for the sample grown at $1 \times 10^{-5}$ Torr.

One of the characteristic features of the 2DEG at the $\mathrm{LaAlO}_{3} / \mathrm{SrTiO}_{3}$ system is thickness dependence of the insulator-metal transition with a critical thickness of $\sim 4$ uc of the $\mathrm{LaAlO}_{3}$ [2]. Sheet conductivity for the $\mathrm{NdAlO}_{3} / \mathrm{SrTiO}_{3}$ interfaces as a function of the number 

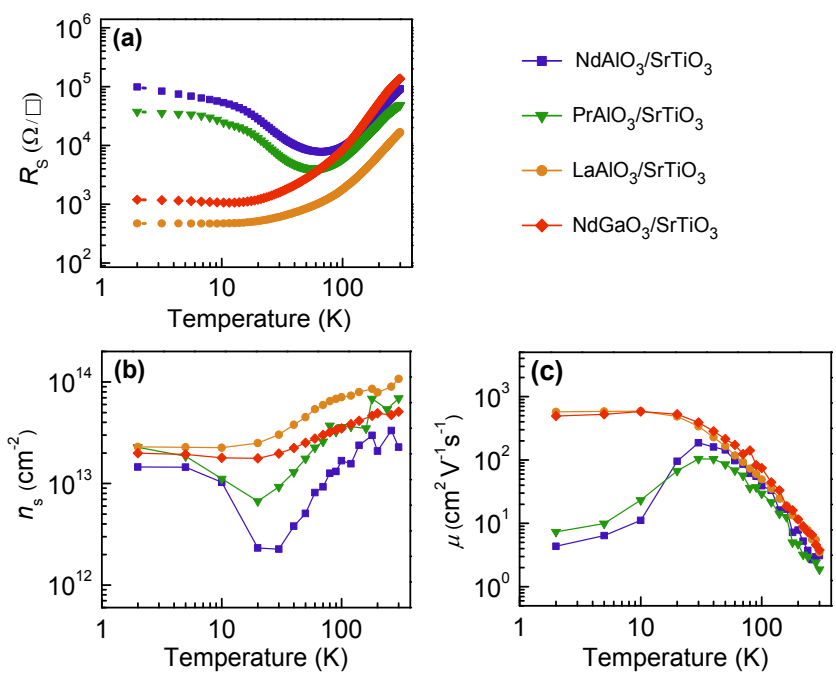

FIG. 3: Comparison of transport properties of different polar/nonpolar $\left(\mathrm{REBO}_{3} / \mathrm{SrTiO}_{3} \mathrm{RE}=\mathrm{La}, \mathrm{Pr}, \mathrm{Nd}, \mathrm{B}=\mathrm{Al}, \mathrm{Ga}\right)$ oxide interfaces. (a) Temperature dependence of $R_{s}$ of the interfaces. Temperature dependence of (b) $n_{s}$ and (c) $\mu$ of the corresponding interfaces.

of unit cells of $\mathrm{NdAlO}_{3}$ is depicted in Fig. 2(d), showing a clear transition from insulating to metallic interface at a thickness around $4 \mathrm{uc}$, with a change in the conductivity of more than three orders of magnitude across the insulator-metal transition. The similarity in the insulator-metal transition to the $\mathrm{LaAlO}_{3} / \mathrm{SrTiO}_{3}$ system confirms the formation of $2 \mathrm{DEG}$ at the $\mathrm{NdAlO}_{3} / \mathrm{SrTiO}_{3}$ interface and supports the idea that the electronic reconstruction could also be the driving mechanism in these interfaces. However, substantial differences observed in temperature dependence of the $n_{s}$ and $\mu$ indicate the effect of the different polar layers.

To further elucidate the polar layer effects a comparison study was carried out and samples with $10 \mathrm{uc}$ $\mathrm{LaAlO}_{3}, \mathrm{PrAlO}_{3}$ and $\mathrm{NdGaO}_{3}$ as the polar over layers were grown at $P_{O 2}=1 \times 10^{-3}$ Torr. Figure 3(a) shows the $R_{s}$ variation with temperature for these various polar/nonpolar combinations. Similar to the case of $\mathrm{NdAlO}_{3} / \mathrm{SrTiO}_{3}, \mathrm{PrAlO}_{3} / \mathrm{SrTiO}_{3}$ interfaces also show upturn in $R_{s}$ at low temperatures. On the other hand, interestingly, $\mathrm{LaAlO}_{3} / \mathrm{SrTiO}_{3}$ and $\mathrm{NdGaO}_{3} / \mathrm{SrTiO}_{3}$ interfaces show a typical metallic behaviour without appreciable upturns in $R_{s}$. Further, their $R_{s}$ at 300 $\mathrm{K}$ appears to have a strong dependence on the $\mathrm{RE}$ cation of the polar layer, where $R_{s}$ is larger $(\sim 90$ $\mathrm{k} \Omega)$ for $\mathrm{NdAlO}_{3}$ compared to that of $\mathrm{LaAlO}_{3}(\sim 20$ $\mathrm{k} \Omega$ ). Figure $3(\mathrm{~b})$ shows the $n_{s}$ variation with temperature; $\mathrm{LaAlO}_{3} / \mathrm{SrTiO}_{3}$ and $\mathrm{NdGaO}_{3} / \mathrm{SrTiO}_{3}$ interfaces show minute carrier freeze-out towards low temperatures compared to $\mathrm{NdAlO}_{3} / \mathrm{SrTiO}_{3}$ and $\mathrm{PrAlO}_{3} / \mathrm{SrTiO}_{3}$, indicating that the interfaces with $\mathrm{LaAlO}_{3}$ and $\mathrm{NdGaO}_{3}$ have less contribution from activated carriers. However, a carrier recovery below $15 \mathrm{~K}$ is observed for the
$\mathrm{NdAlO}_{3} / \mathrm{SrTiO}_{3}$ and $\mathrm{PrAlO}_{3} / \mathrm{SrTiO}_{3}$ interfaces, which is possibly due to the difference transport mechanism in this low temperature localized regime (further investigation is needed to understand this unusual behaviour). The most remarkable effects are seen in temperature dependence of mobility which is shown in Fig. 3(c). For $\mathrm{NdAlO}_{3} / \mathrm{SrTiO}_{3}$ and $\mathrm{PrAlO}_{3} / \mathrm{SrTiO}_{3}$ interfaces, $\mu$ increases initially with decreasing temperature and drops dramatically for temperatures below $40 \mathrm{~K}$. On the other hand interfaces with $\mathrm{LaAlO}_{3}$ and $\mathrm{NdGaO}_{3}$ show an increase in $\mu$ and tend to saturate at low temperatures. Interesting observation is that even though the $n_{s}(2 \mathrm{~K})$ is nearly the same $\left(\sim 2 \times 10^{13} \mathrm{~cm}^{-2}\right)$ for all the interfaces, $R_{s}$ and $\mu$ exhibit significant divergence, implying that the polar layer has a significant influence on the charge carriers at the polar/nonpolar interface.

Despite these polar layers appear similar in nature, yet there are few differences. Firstly, these polar layers comprise of different chemical elements at A-site (RE cation) and $\mathrm{B}$-site in the perovskite $\left(\mathrm{ABO}_{3}\right)$ structure. When the polar layer $\left(\mathrm{REBO}_{3}\right)$ is deposited on top of the $\mathrm{TiO}_{2}$ terminated $\mathrm{SrTiO}_{3}(100)$, the first unit cell at the interface on either side can be viewed as a stack of $\mathrm{REO} / \mathrm{TiO}_{2} / \mathrm{SrO}$ layers. In this picture, across the interface $\mathrm{TiO}_{2}$ layer will have a different electronic environment in presence of different RE cations of polar layer. Secondly, different polar layers cause different lattice mismatch with $\mathrm{SrTiO}_{3}$ which in turn create a different interfacial strain. To further understand these effects, we plotted the A-site cationic dependence of the $n_{s}$ for corresponding interfaces as shown in Fig. 4(b). Clearly $n_{s}(300 \mathrm{~K})$ are high in the case of La based interface and lower for Nd based interfaces, similar to the case of $\mathrm{SrTiO}_{3} / \mathrm{REO} / \mathrm{SrTiO}_{3}$ heterostructures reported [20]. However, $n_{s}$ at $2 \mathrm{~K}$ have rather small dependency on the RE cation with $n_{s}$ is of the order of $1-2 \times 10^{13} \mathrm{~cm}^{-2}$ for all interfaces. The RE cation dependency of $n_{s}$ marks the presence of strong electronic correlation of interface with polar layers.

Now we comment on mobility. Referring back to Fig. 3(c), the $\mu$ drop was only observed in the case of $\mathrm{NdAlO}_{3} / \mathrm{SrTiO}_{3}$ and not in the $\mathrm{NdGaO}_{3} / \mathrm{SrTiO}_{3}(\mathrm{Nd}$ is A-site cation for both cases), implying that we cannot attribute this $\mu$ drop to the proximity effects of $\mathrm{RE}$ ions at the interface. So the only other difference these polar layers offer is the lattice mismatch at the interface with the $\mathrm{SrTiO}_{3}$ substrate. Lattice mismatch here is estimated by considering in-plane lattice parameters (a schematic diagram is shown in Fig. 4(a)), among them the $\mathrm{NdGaO}_{3} / \mathrm{SrTiO}_{3}$ interface offers the least lattice mismatch $(\sim 1.6 \%)$ and $\mathrm{NdAlO}_{3} / \mathrm{SrTiO}_{3}$ the largest $(\sim 3.6 \%)$. Figure $4(\mathrm{c})$ shows the $\mu$ variation with lattice mismatch; here $\mu$ (300 K) shows modest dependency to the lattice mismatch with values typically of the order of $1-5 \mathrm{~cm}^{2} \mathrm{~V}^{-1} \mathrm{~s}^{-1}$, which suggests the predominant electron-phonon scattering at $300 \mathrm{~K}$. In contrast, $\mu(2 \mathrm{~K})$ shows strong dependence on lattice mismatch: $\mu$ is $\sim 500$ $\mathrm{cm}^{2} \mathrm{~V}^{-1} \mathrm{~s}^{-1}$ for $\mathrm{NdGaO}_{3} / \mathrm{SrTiO}_{3}$ and only $4 \mathrm{~cm}^{2} \mathrm{~V}^{-1} \mathrm{~s}^{-1}$ for $\mathrm{NdAlO}_{3} / \mathrm{SrTiO}_{3}$ (a drop of a factor of 100). It ap- 

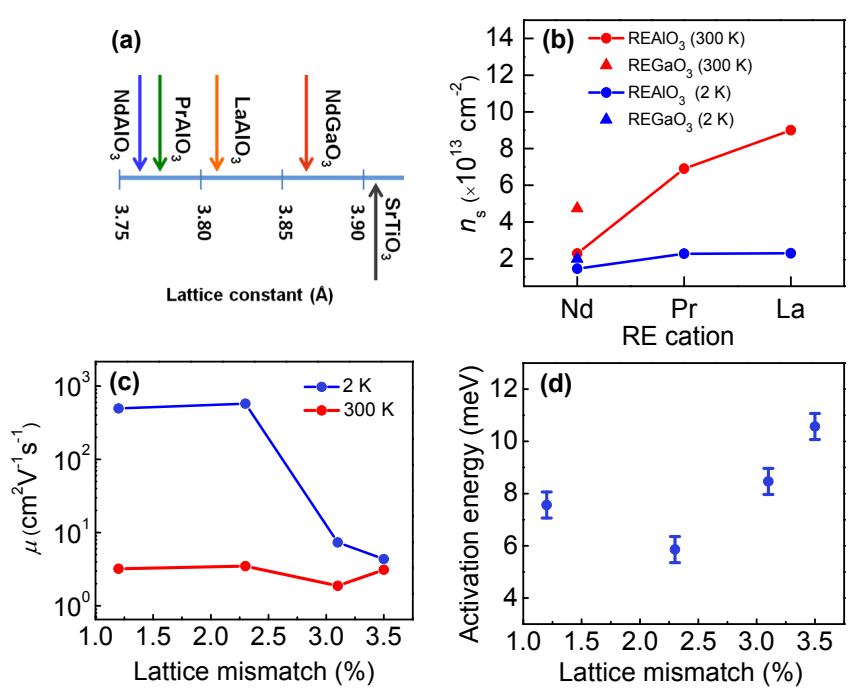

FIG. 4: (a) Schematic diagram representing lattice parameters of different polar oxides and $\mathrm{SrTiO}_{3}$ is also shown. (b) $n_{s}$ dependency on RE cation of polar layer at $300 \mathrm{~K}$ and 2 K. (c) $\mu$ of different interfaces under lattice mismatch at 300 and $2 \mathrm{~K}$. (d) Variation in activation energy as a function of lattice mismatch.

pears that the large lattice mismatch is limiting the $\mu$. We note here that even though the carrier density $\left(n_{s}\right)$ at $2 \mathrm{~K}$ is almost the same $\left(2 \times 10^{13} \mathrm{~cm}^{-2}\right)$ for all interfaces but a large variation in $\mu$ is observed, indicating strong electronic correlations among carriers. Thus, we argue that the combined effects of strain and strong electron correlation are crucial in controlling the $\mu$. Further, because of a smaller lattice mismatch a higher $\mu$ is expected in the case of $\mathrm{NdGaO}_{3}(\sim 1.6 \%)$ compared to the case of $\mathrm{LaAlO}_{3}$ (mismatch $\sim 2.3 \%$ ) due to lesser mismatch, however the mobility values are just comparable in both cases. We attribute this to the residual impurity scattering in $\mathrm{SrTiO}_{3}$ itself.

As mentioned earlier a large carrier freeze out is observed for the carriers with temperature. To examine this, we extracted activation energy from Arrhenius plots (between 20 and $300 \mathrm{~K}$ ) of the $n_{s}$ versus temperature graphs in Fig. 3(b). Activation energies of the order of few $\mathrm{meV}$ are in agreement with the previous report on $\mathrm{LaAlO}_{3} / \mathrm{SrTiO}_{3}$ [19]. Figure 4(d) shows activation en- ergy as a function of lattice mismatch. Interestingly, activation energy gradually increases with lattice mismatch and it is largest $(16 \mathrm{meV})$ for the $\mathrm{NdAlO}_{3} / \mathrm{SrTiO}_{3}$. In general, activation energy indicates the relative position of carrier donor level from the conduction band in energy scale. Its variation with lattice mismatch may suggest relative shift in position of donor level among these interfaces. Recently, it has been proposed that strain could leads to distortion of $\mathrm{Ti}$ octahedra through octahedral rotation/tilt in $\mathrm{SrTiO}_{3}$ based heterostructures $[20,21]$, which in turn could alter the position of energy levels. Since electronic effects are quite sensitive to the degree of octahedral distortions, it is further interesting to study the effects of octahedral distortions on novel phases at these interfaces.

\section{CONCLUSION}

In conclusion, we demonstrate the observation of $2 \mathrm{DEG}$ at the $\mathrm{NdAlO}_{3} / \mathrm{SrTiO}_{3}, \mathrm{PrAlO}_{3} / \mathrm{SrTiO}_{3}$ and $\mathrm{NdGaO}_{3} / \mathrm{SrTiO}_{3}$ interfaces and suggest that polar discontinuity could be the prime origin of conductivity at these polar/nonpolar interfaces. We showed that the combined effects of strong correlations and interface strain offered by polar layers predominantly control the carrier density and mobility of the 2DEG. Moreover the presence of large octahedral distortions due to interface strain may play an important role in manipulating the novel phases at the interface. Our observations further emphasize the key role of polar layers in these heterostructures and may provide an opportunity to tune the properties at selected polar/nonpolar oxide interfaces.

\section{Acknowledgments}

We thank the National Research Foundation (NRF) Singapore under the Competitive Research Program (CRP) "Tailoring Oxide Electronics by Atomic Control" NRF2008NRF-CRP002-024, National University of Singapore (NUS) cross-faculty grant and FRC (ARF Grant No. R-144-000-278-112) for financial support.
* Email: ariando@nus.edu.sg

1 A. Ohtomo and H. Y. Hwang, Nature 427, 423 (2004).

2 S. Thiel, G. Hammerl, A. Schmehl, C. W. Schneider, and J. Mannhart, Science 313, 1942 (2006).

${ }^{3}$ N. Reyren, S. Theil, A. D. Caviglia, L. Fitting Kourkoutis, G. Hammer, C. Richter, C. W. Schneider, T. Kopp, A. S. Ruetschi, D. Jaccard, M. Gabay, D. A. Muller, J. -M. Triscone, and J. Mannhart, Science 317, 1196 (2007).

4 A. Brinkman, M. Huijben, M. Van Zalk, J. Huijben, U.
Zeitler, J. C. Maan, W. G. Van der Wiel, G. Rijinders, D. H. A. Blank, and H. Hilgenkamp, Nature Mater. 6, 493 (2007).

5 Ariando, X. Wang, G. Baskaran, Z. Q. Liu, J. Huijben, J. B. Yi, A. Annadi, A. Roy Barman, A. Rusydi, S. Dhar, Y. P. Feng, J. Ding, H. Hilgenkamp, and T. Venkatesan, Nat. Commun. 2, 188 (2011).

${ }^{6}$ C. Cen, S. Thiel, G. Hammerl, C. W. Schneider, K. E. Andersen, C. S. Hellberg, J. Mannhart, and J. Levy, Nature 
Mater. 7, 298 (2008).

7 Y. Xie, Y. Hikita, C. Bell, and H. Y. Hwang, Nat. Commun. 2, 494 (2011).

8 C. W. Bark. D. A. Felker, Y. Wang, Y. Zhang, H. W. Jang, C. M. Folkman, J. W. Park, S. H. Baek, H. Zhou, D. D. Fong, X. Q. Pan, E. Y. Tsymbal, M. S. Rzchowski, and C. B. Eom, PNAS 108, 12, 4720 (2011).

9 C. Cancellieri, D. Fontaine, S. Gariglio, N. Reyren, A. D. Caviglia, A. Fete, S. J. Leake, S. A. Pauli, P. R. Willmott, M. Stengel, Ph. Ghosez, and J.-M. Triscone, Phys. Rev. Lett. 107, 056102 (2011).

10 C. W. Bark, P. Sharma, Y. Wang, S.H. Baek, S. Lee, S. Ryu, C. M. Folkman, T. R.Paudel, A. Kumar, S.V. Kalinin, A. Sokolov, E. Y. Tsymbal, M. S. Rzchowski, A.Gruverman, and C.B. Eom, Nano Lett. DOI: 10.1021/nl3001088.

11 P. Perna, D. Maccariello, M. Radovic, U. Scotti di Uccio, I. Pallecchi, M. Codda, D. Marr, C. Cantoni, J. Gazquez, M. Varela, S. J. Pennycook, and F. Miletto Granozio, Appl. Phys. Lett. 97, 152111 (2010).

12 N. Nakagawa, H. Y. Hwang, and D. A. Muller, Nature Mater. 5, 204 (2006).

13 M. Kawasaki, K. S. Takahashi, T. Maeda, R. Tsuchiya, M. Shinohara, O. Ishiyama, T. Yonezawa, M. Yoshimoto, and H. Koinuma, Science 266, 1540 (1994).
14 G. Koster, B. L. Kropman, G. J. H. M. Rijinders, D. H. A. Blank, and H. Rogalla, Appl. Phys. Lett.73, 2920 (1998).

15 C. Bell, S. Harashima, Y. Hikita, and H. Y. Hwang, Appl. Phys. Lett. 94, 222111 (2009).

16 W. Siemons, G. Koster, H. Yamamoto,T. H. Geballe, D. H. A. Blank, and M. R. Beasley, Phys. Rev. B 76, 155111 (2007).

17 A. Kalabukhov, R. Gunnarsson, J. Borjesson, E. Olsson, T. Claeson, and D. Winkler, Phys. Rev. B 75, 121404 (R) (2007).

18 M. Huijben, G. Koster, H.J.A. Molegraaf, M.K. Kruize, S. Wenderich, J.E. Kleibeuker, A. McCollam, V.K. Guduru, A. Brinkman, H. Hilgenkamp, U. Zeitler, J.C. Maan, D.H.A. Blank, and G. Rijnders, ArXiv :1008.1896v1.

19 M. Huijben, G. Rijinders, D. H. A. Blank,S. Bals, S. V. Aert, J. Verbeeck, G. V. Tendeloo, A. Brinkman, and H. Hilgenkamp, Nature Mater. 5, 556 (2006).

20 H.W. Jang, D. A. Felker, C. W. Bark, Y. Wang, M. K. Niranjan, C. T. Nelson, Y. Zhang, D. Su, C. M. Folkman, S. H. Baek, S. Lee, K. Janicka, Y. Zhu, X. Q. Pan, D. D. Fong, E. Y. Tsymbal, M. S. Rzchowski, and C. B. Eom, Science 331, 886 (2011).

21 C. L. Jia, S. B. Mi, M. Faley, U. Poppe, J. Schubert, and K. Urban, Phys. Rev. B 79, 081405(R) (2009). 